\title{
Semi-Compatible Maps On Intuitionistic Fuzzy Metric Space
}

\author{
Pradeep Kumar Dwivedi ${ }^{1} \&$ Anil Rajput ${ }^{2}$ \\ ${ }^{I}$ Millennium Group of Institutions, Bhopal, India, \\ ${ }^{2}$ CSA, Govt. PG College, Sehore, India,
}

\begin{abstract}
In this paper, we prove common fixed point theorem for semi-compatible mappings on intuitionistic fuzzy metric space with different some conditions of Park and Kim ([10], 2008). This research extended and generalized the results of Singh and Chauhan ([14], 2000).

The concept of fuzzy set was developed extensively by many authors and used in various fields. Several authors have defined fuzzy metric space Kramosil and Michalek(([5],1975) etc.) with various methods to use this concept in analysis. Jungck (([3],1986), ([4],1988)) researched the more generalized concept compatibility than commutativity and weak commutativity in metric space and proved common fixed point theorems, and Singh and Chauhan ([14],2000) introduced the concept of compatibility in fuzzy metric space and studied common fixed point theorems for four compatible mappings.

Recently, Park et. al. ([7], 2006) defined the upgraded intuitionistic fuzzy metric space and Park et.al. (([8], 2008), ([9], 1999), ([11], 2007), ([12], 2005)) studied several theories in this space. Also, Park and Kim ([10], 2008) proved common fixed point theorem for self maps in intuitionistic fuzzy metric space.
\end{abstract}

\section{Introduction:}

In this paper, we prove common fixed point theorem for semi-compatible mappings on intuitionistic fuzzy metric space with different some conditions of Park and Kim ([10], 2008). This research extended and generalized the results of Singh and Chauhan ([14], 2000).

We give some definitions and properties of intuitionistic fuzzy metric space. Throughout this paper, $\tilde{\mathrm{N}}$ will denote the set of all positive integers.

Let us recall Schweizer and Sklar (see $([13], 1960)$ ) that a continuous t-norm is a binary operation* :

$[0,1] \times[0,1] \rightarrow[0,1]$ which satisfies the following conditions:

(a) * is commutative and associative;

(b) * is continuous;

(c) $\mathrm{a} * 1=\mathrm{a}$ for all $\mathrm{a} \in[0,1]$;

(d) $\quad \mathrm{a} * \mathrm{~b} \leq \mathrm{c} * \mathrm{~d}$ whenever $\mathrm{a} \leq \mathrm{c}$ and $\mathrm{b} \leq \mathrm{d}(\mathrm{a}, \mathrm{b}, \mathrm{c}, \mathrm{d} \in[0,1])$.

Similarly, a continuous t-conorm is a binary operation $\diamond:[0,1] \times[0,1] \rightarrow[0,1]$ which satisfies the following conditions :

(a) $\diamond$ is commutative and associative;

(b) $\diamond$ is continuous;

(c) a $\diamond 0=$ a for all $\mathrm{a} \in[0,1]$;

(d) a $\diamond \mathrm{b} \geq \mathrm{c} \diamond \mathrm{d}$ whenever $\mathrm{a} \leq \mathrm{c}$ and $\mathrm{b} \leq \mathrm{d}(\mathrm{a}, \mathrm{b}, \mathrm{c}, \mathrm{d} \in[0,1])$.

Also, let us recall (see [6] that the following conditions are satisfied :

(a) For any any $r_{1}, r_{2} \in(0,1)$ with $r_{1}>r_{2}$ there exist $r_{3}, r_{4} \in(0,1)$ such that $r_{1} * r_{3} \geq r_{2}$ and $r_{4} \diamond r_{2} \leq r_{1}$;

(b) For any $r_{5} \in(0,1)$, there exist $r_{6}, r_{7} \in(0,1)$ such that $r_{6} * r_{6} \geq r_{5}$

and $r_{7} \diamond r_{7} \leq r_{5}$.

1.1 Definition:- (Park and Kwun ([7], 2006)). The 5-tuple (X, M, N, *, $\diamond$ ) is said to be an intuitionistic fuzzy metric space if $\mathrm{X}$ is an arbitrary set, $*$ is a continuous t-norms, $\diamond$ is a continuous t-conorm and $\mathrm{M}, \mathrm{N}$ are fuzzy sets on $\mathrm{X}^{2} \mathrm{x}(0, \infty)$ satisfying the following conditions; for all $x, \mathrm{y}, \mathrm{z} \in X$, such that -

(a) $\mathrm{M}(x, \mathrm{y}, \mathrm{t})>0$,

(b) $\mathrm{M}(x, \mathrm{y}, \mathrm{t})=1 \Leftrightarrow x=\mathrm{y}$,

(c) $\mathrm{M}(x, \mathrm{y}, \mathrm{t})=\mathrm{M}(\mathrm{y}, x, \mathrm{t})$

(d) $\mathrm{M}(x, \mathrm{y}, \mathrm{t}) * \mathrm{M}(\mathrm{y}, \mathrm{z}, \mathrm{s}) \leq \mathrm{M}(x, \mathrm{z}, \mathrm{t}+\mathrm{s})$,

(e) $\mathrm{M}(x, \mathrm{y},):.(0, \infty) \rightarrow(0,1]$ is continuous,

(f) $\mathrm{N}(x, \mathrm{y}, \mathrm{t})>0$

(g) $\mathrm{N}(x, \mathrm{y}, \mathrm{t})=0 \Leftrightarrow x=\mathrm{y}$,

(h) $\mathrm{N}(x, \mathrm{y}, \mathrm{t})=\mathrm{N}(\mathrm{y}, x, \mathrm{t})$, 
(i) $\mathrm{N}(x, \mathrm{y}, \mathrm{t}) \diamond \mathrm{N}(\mathrm{y}, \mathrm{z}, \mathrm{s}) \geq \mathrm{N}(x, \mathrm{z}, \mathrm{t}+\mathrm{s})$,

(j) $\mathrm{N}(x, \mathrm{y},):.(0, \infty) \rightarrow(0,1]$ is continuous.

Note that $(M, N)$ is called an intuitionistic fuzzy metric on X. The functions

$\mathrm{M}(x, \mathrm{y}, \mathrm{t})$ and $\mathrm{N}(x, \mathrm{y}, \mathrm{t})$ denote the degree of nearness and the degree of non-nearness between $x$ and $y$ with respect to t, respectively.

1.2 Definition:- (Park and Kwun ([12], 2005)). Let X be an intuitionistic fuzzy metric space. Then (a) A sequence $\left\{x_{\mathrm{n}}\right\} \subset \mathrm{X}$ is convergent to $x$ in $\mathrm{X}$ if and only if for each $\varepsilon>0, \mathrm{t}>0$, there exists $\mathrm{n}_{0} \in \tilde{\mathrm{N}}$ such that $\mathrm{M}(x$ $\left.{ }_{\mathrm{n}}, x, \mathrm{t}\right)>1-\varepsilon, \mathrm{N}\left(x_{\mathrm{n}}, x, \mathrm{t}\right)<\varepsilon$ for all $\mathrm{n} \geq \mathrm{n}_{0}$.

(b) A sequence $\left\{x_{\mathrm{n}}\right\} \subset \mathrm{X}$ is called Cauchy sequence if and only if for each $\varepsilon>0$, t $>0$, there exists $\mathrm{n}_{0} \in \tilde{\mathrm{N}}$ such that $\mathrm{M}\left(x_{\mathrm{n}}, x_{\mathrm{m}}, \mathrm{t}\right)>1-\varepsilon, \mathrm{N}\left(x_{\mathrm{n}}, x_{\mathrm{m}}, \mathrm{t}\right)<\varepsilon$ for all $\mathrm{n}, \mathrm{m} \geq \mathrm{n}_{0}$

(c) $\mathrm{X}$ is complete if every Cauchy sequence in $\mathrm{X}$ is convergent.

1.3 Definition:- (Park and Kim ([10], 2008)). Let A, B be mappings from intuitionistic fuzzy metric space X into itself.

(a) (A, B) are said to be compatible if and only if

$\lim _{n \rightarrow \infty} \mathrm{M}\left(\mathrm{AB} x_{\mathrm{n}}, \mathrm{BA} x_{\mathrm{n}}, \mathrm{t}\right)=1, \lim _{n \rightarrow \infty} \mathrm{N}\left(\mathrm{AB} x_{\mathrm{n}}, \mathrm{BA} x_{\mathrm{n}}, \mathrm{t}\right)=0$, for all $\mathrm{t}>0$, whenever $\left\{x_{\mathrm{n}}\right\} \subset \mathrm{X}$ such that $\lim n \rightarrow \infty \mathrm{A} x_{\mathrm{n}}=\lim n \rightarrow \infty \mathrm{B} x_{\mathrm{n}}=x$ for some $x \in \mathrm{X}$.

(b) (A, B) are said to be semi compatible if and only if

$\lim _{n \rightarrow \infty} \mathrm{M}\left(\mathrm{AB} x_{\mathrm{n}}, \mathrm{B} x, \mathrm{t}\right)=1, \lim _{n \rightarrow \infty} \mathrm{N}\left(\mathrm{AB} x_{\mathrm{n}}, \mathrm{B} x, \mathrm{t}\right)=0$, for all $\mathrm{t}>0$, whenever $\left\{x_{\mathrm{n}}\right\} \subset \mathrm{X}$ such that $\lim n \rightarrow \infty \mathrm{A} x_{\mathrm{n}}=\lim n \rightarrow \infty \mathrm{B} x_{\mathrm{n}}=x$ for some $x \in \mathrm{X}$.

1.4 Lemma:- (Park[10],2008)). Let A, B to be self mappings on intuitionistic fuzzy metric space X. If B is continuous, then $(\mathrm{A}, \mathrm{B})$ is semi-compatible if and only if $(\mathrm{A}, \mathrm{B})$ is compatible.

\section{Main Result}

2.1 Theorem:- Let $\mathrm{P}, \mathrm{Q}, \mathrm{S}$ and $\mathrm{T}$ be self maps of complete intuitionistic fuzzy metric space $\mathrm{X}$ with $\mathrm{t}$ - norm*and $\mathrm{t}-$ conorms (defined by $\mathrm{a}^{*} \mathrm{~b}=\min \{\mathrm{a}, \mathrm{b}\}$ and $\mathrm{a}(\mathrm{b}=\max \{\mathrm{a}, \mathrm{b}\}, \mathrm{a}, \mathrm{b} \in[0,1]$, satisfying

(a) $(\mathrm{P}, \mathrm{S})$ and $(\mathrm{Q}, \mathrm{T})$ are semi-compatible pairs of maps,

(b) $\mathrm{S}$ and $\mathrm{T}$ are continuous,

(c) $\mathrm{P}^{\mathrm{p}}(\mathrm{x}) \subset \mathrm{T}^{\mathrm{t}}(\mathrm{x}), \quad \mathrm{Q}^{\mathrm{q}}(\mathrm{x}) \subset \mathrm{S}^{\mathrm{s}}(\mathrm{X})$

(d) $\mathrm{M}\left(\mathrm{P}^{\mathrm{p}} x, \mathrm{Q}^{\mathrm{q}} \mathrm{y}, \mathrm{kt}\right) \geq \operatorname{Min}\left\{\mathrm{M}\left(S_{x}^{s}, T_{y}^{t}, t\right), M\left(P_{x}^{p}, S_{x}^{s}, t\right)\right.$,

$\mathrm{M}\left(Q_{y}^{q}, T_{y}^{t}, t\right), M\left(P_{x}^{p}, T_{y}^{t}, \alpha t\right)$,

$\left.\mathrm{M}\left(Q_{y}^{q}, S_{x}^{s},(2-\alpha) t\right)\right\}$,

$\mathrm{N}\left(P_{x}^{p}, Q_{y}^{q}, k t\right) \leq \max \left\{N\left(S_{x}^{s}, T_{y}^{t}, t\right), N\left(P_{x}^{p}, S_{x}^{s}, t\right)\right.$,

$\mathrm{N}\left(Q_{y}^{q}, T_{y}^{t}, t\right), N\left(P_{x}^{p}, T_{y}^{t}, \alpha t\right)$,

$\left.\mathrm{N}\left(Q_{y}^{q}, S_{x}^{s},(2-\alpha) t\right)\right\}$.

(e)

$$
\lim t \rightarrow \infty \mathrm{M}(x, \mathrm{y}, \mathrm{t})=1,
$$

$\lim t \rightarrow \infty \mathrm{N}(x, \mathrm{y}, \mathrm{t})=0$

for all $x, \mathrm{y} \in \mathrm{x}, \alpha \in(0,2), \mathrm{t}>0$ and $\mathrm{p}, \mathrm{q}, \mathrm{s}, \mathrm{t} \in \tilde{\mathrm{N}}$.

Then $\mathrm{P}, \mathrm{Q}, \mathrm{S}$ and $\mathrm{T}$ have a unique common fixed point in $\mathrm{X}$.

Proof. Let $x_{\mathrm{o}}$ be an arbitrary point in $\mathrm{X}$. we can inductively construct a sequence $\left\{\mathrm{y}_{\mathrm{n}}\right\} \subset X$ such that $\mathrm{y}_{2 \mathrm{n}-1}=\mathrm{T}^{\mathrm{t}} x_{2 \mathrm{n}-1}=\mathrm{P}^{\mathrm{p}} x_{2 \mathrm{n}-2,}, \mathrm{y}_{2 \mathrm{n}}=\mathrm{S}^{\mathrm{s}} x_{2 \mathrm{n}}=\mathrm{Q}^{\mathrm{q}} x_{2 \mathrm{n}-1}$ for $\mathrm{n}=1,2,3, \ldots$

First, we prove that $\left\{\mathrm{y}_{\mathrm{n}}\right\}$ is a Cauchy sequence, from (d) with $\alpha=1$, we have.

$M\left(\mathrm{y}_{2 \mathrm{n}+1}, \mathrm{y}_{2 \mathrm{n}+2}, \mathrm{Kt}\right)=\quad \mathrm{M}\left(\mathrm{P}^{\mathrm{p}}{ }_{x 2 \mathrm{n}}, \mathrm{Q}_{x 2 \mathrm{n}+1}^{\mathrm{q}}, \mathrm{Kt}\right)$

$\geq \min \quad\left\{\mathrm{M}\left(\mathrm{S}_{x 2 \mathrm{n}}^{\mathrm{s}}, \mathrm{T}_{x 2 \mathrm{n}+1}^{\mathrm{t}}, \mathrm{t}\right), \mathrm{M}\left(\mathrm{P}_{x 2 \mathrm{n}}^{\mathrm{p}}, \mathrm{S}_{x 2 \mathrm{n}}^{\mathrm{s}}, \mathrm{t}\right)\right.$,

$\mathrm{M}\left(\mathrm{Q}_{x 2 \mathrm{n}+1}^{\mathrm{q}}, \mathrm{T}_{x 2 \mathrm{n}+1}^{\mathrm{t}}, \mathrm{t}\right), \mathrm{M}\left(\mathrm{P}_{x 2 \mathrm{n}}^{\mathrm{p}}, \mathrm{T}_{x 2 \mathrm{n}+1}^{\mathrm{t}}, \mathrm{t}\right)$,

$\left.\mathrm{M}\left(\mathrm{Q}_{x 2 \mathrm{n}+1}^{\mathrm{q}}, \mathrm{S}_{x 2 \mathrm{n}}^{\mathrm{s}}, \mathrm{t}\right)\right\}$

$\geq \operatorname{Min} \quad\left\{M\left(y_{2 n}, y_{2 n+1}, t\right), M\left(y_{2 n+1}, y_{2 n}, t\right), M\left(y_{2 n+2}, y_{2 n+1}, t\right)\right.$,

$$
\left.M\left(y_{2 n+1}, y_{2 n+1}, t\right), M\left(y_{2 n+2}, y_{2 n}, t\right)\right\}
$$

$\geq \operatorname{Min} \quad\left\{M\left(y_{2 n}, y_{2 n+1}, t\right), M\left(y_{2 n+2}, y_{2 n+1}, t\right), 1\right\}$

$\mathrm{N}\left(\mathrm{y}_{2 \mathrm{n}+1}, \mathrm{y}_{2 \mathrm{n}+2}, \mathrm{Kt}\right)=\quad\left(\mathrm{P}_{x 2 \mathrm{n}}^{\mathrm{p}}, \mathrm{Q}^{\mathrm{q}} \mathrm{x}_{2 \mathrm{n}+1}, \mathrm{Kt}\right)$ 


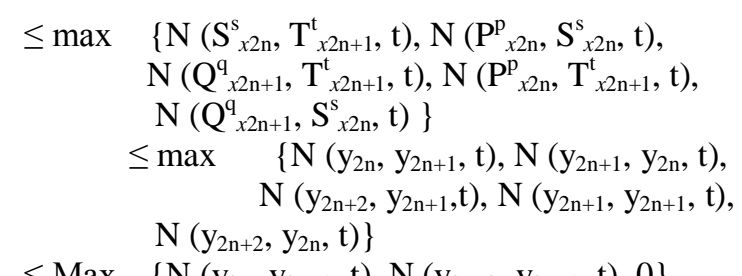

$\leq \operatorname{Max} \quad\left\{N\left(y_{2 n}, y_{2 n+1}, t\right), N\left(y_{2 n+2}, y_{2 n+1}, t\right), 0\right\}$

which implies

$M\left(y_{2 n+1}, y_{2 n+2}, k t\right) \geq M\left(y_{2 n}, y_{2 n+1}, t\right)$,

$N\left(y_{2 n+1}, y_{2 n+2}, k t\right) \leq N\left(y_{2 n}, y_{2 n+1}, t\right)$,

Generally, $M\left(y_{n}, y_{n+1}, k t\right) \geq M\left(y_{n-1}, y_{n}, t\right)$,

$N\left(y_{n}, y_{n+1}, k t\right) \leq N\left(y_{n-1}, y_{n}, t\right)$.

Therefore,

$$
\begin{aligned}
\mathrm{M}\left(\mathrm{y}_{\mathrm{n}}, \mathrm{y}_{\mathrm{n}+1}, \mathrm{t}\right) & \geq \mathrm{M}\left(\mathrm{y}_{\mathrm{n}-1}, \mathrm{y}_{\mathrm{n}}, \frac{t}{k}\right) \\
& \geq \ldots \\
& \geq \mathrm{M}\left(\mathrm{y}_{0}, \mathrm{y}_{1}, \frac{t}{k^{n}}\right)
\end{aligned}
$$

Taking limit $\mathrm{n} \rightarrow \infty$ then it tends to $\rightarrow 1$ as

$$
\begin{aligned}
\mathrm{N}\left(\mathrm{y}_{\mathrm{n}}, \mathrm{y}_{\mathrm{n}+1}, \mathrm{t}\right) & \leq \mathrm{N}\left(\mathrm{y}_{\mathrm{n}-1}, \mathrm{y}_{\mathrm{n}}, \frac{t}{k}\right) \\
& \leq \ldots \\
& \leq \mathrm{N}\left(\mathrm{y}_{0}, \mathrm{y}_{1}, \frac{t}{k^{n}}\right) \quad \rightarrow 0 \text { as } \mathrm{n} \rightarrow \infty
\end{aligned}
$$

Hence for $\mathrm{t}>0$ and $\varepsilon \in(0,1)$, we can choose $\mathrm{n}_{\mathrm{o}} \in \tilde{\mathrm{N}}$ such that

for all $\mathrm{n} \geq \mathrm{n}_{\mathrm{o}}$.

$$
\mathrm{M}\left(\mathrm{y}_{\mathrm{n}}, \mathrm{y}_{\mathrm{n}+1}, \mathrm{t}\right)>1-\varepsilon, \mathrm{N}\left(\mathrm{y}_{\mathrm{n}}, \mathrm{y}_{\mathrm{n}+1}, \mathrm{t}\right)<\varepsilon
$$

Suppose that for $\mathrm{m}$,

for all $\mathrm{n} \geq \mathrm{n}_{\mathrm{o}}$ and for every $\mathrm{m} \in \tilde{\mathrm{N}}$.

$$
M\left(y_{n}, y_{n+m}, t\right)>1-\varepsilon, N\left(y_{n}, y_{n+m}, t\right)<\varepsilon
$$

Then

$$
\mathrm{M}\left(\mathrm{y}_{\mathrm{n}}, \mathrm{y}_{\mathrm{n}+\mathrm{m}+1}, \mathrm{t}\right) \geq \min \left\{\mathrm{M}\left(\mathrm{y}_{\mathrm{n}}, \mathrm{y}_{\mathrm{n}+\mathrm{m}}, \frac{t}{2}\right), \mathrm{M}\left(\mathrm{y}_{\mathrm{n}+\mathrm{m}}, \mathrm{y}_{\mathrm{n}+\mathrm{m}+1}, \frac{t}{2}\right)\right\}
$$

$>1-\varepsilon$,

$$
\begin{aligned}
& \mathrm{N}\left(\mathrm{y}_{\mathrm{n}}, \mathrm{y}_{\mathrm{n}+\mathrm{m}+1}, \mathrm{t}\right) \leq \max \left\{\mathrm{N}\left(\mathrm{y}_{\mathrm{n}}, \mathrm{y}_{\mathrm{n}+\mathrm{m}}, \frac{t}{2}\right), \mathrm{N}\left(\mathrm{y}_{\mathrm{n}+\mathrm{m}}, \mathrm{y}_{\mathrm{n}+\mathrm{m}+1}, \frac{t}{2}\right)\right\} \\
& <\varepsilon .
\end{aligned}
$$

Therefore $\left\{\mathrm{y}_{\mathrm{n}}\right\} \subset \mathrm{X}$ is a cauchy sequence.

Second, we prove that $\mathrm{P}^{\mathrm{p}}, \mathrm{Q}^{\mathrm{q}}, \mathrm{S}^{\mathrm{S}}$, and $\mathrm{T}^{\mathrm{t}}$ have a unique common fixed point.

Since $\left\{\mathrm{y}_{\mathrm{n}}\right\}$ converges to some point $x$ from completeness of $\mathrm{X}$,

Since $\mathrm{S}$ is continuous, hence

$$
\mathrm{P}^{\mathrm{p}} x_{2 \mathrm{n}} \rightarrow x, S^{S} x_{2 \mathrm{n} \rightarrow} x, \mathrm{Q}^{\mathrm{q}}{ }_{x 2 \mathrm{n}-1 \rightarrow} x \text { and } \mathrm{T}_{\mathrm{x} 2 \mathrm{n}-1 \rightarrow}^{\mathrm{t}} x
$$

$$
\mathrm{S}^{\mathrm{s}}\left(\mathrm{P}^{\mathrm{p}} x_{2 \mathrm{n}}\right) \rightarrow \mathrm{S}^{\mathrm{S}}(x)
$$

Thus for $\mathrm{t}>0$ and $\varepsilon \in(0,1)$, there exists an $\mathrm{n}_{\mathrm{o}} \in \tilde{\mathrm{N}}$ such that

$$
\begin{aligned}
& \mathrm{M}\left(\mathrm{S}^{\mathrm{s}}\left(\mathrm{P}^{\mathrm{p}} x_{2 \mathrm{n}}\right), \mathrm{S}^{\mathrm{s}}(x), \frac{t}{2}\right)>1-\varepsilon, \\
& \mathrm{N}\left(\mathrm{S}^{\mathrm{s}}\left(\mathrm{P}^{\mathrm{p}} x_{2 \mathrm{n}}\right), \mathrm{S}^{\mathrm{s}}(x), \frac{t}{2}\right)<\varepsilon
\end{aligned}
$$

for all $\mathrm{n} \geq \mathrm{n}_{\mathrm{o}}$. Also since $(\mathrm{P}, \mathrm{S})$ and $(\mathrm{Q}, \mathrm{T})$ are semi - compatible pairs, by Lemma 1.4, $(\mathrm{P}, \mathrm{S})$ and $(\mathrm{Q}, \mathrm{T})$ are compatible pairs.

Therefore $\left(\mathrm{P}^{\mathrm{p}}, \mathrm{S}^{\mathrm{s}}\right)$ and $\left(\mathrm{Q}^{\mathrm{q}}, \mathrm{T}^{\mathrm{t}}\right)$ are compatible pairs for all $\mathrm{P}, \mathrm{q}, \mathrm{s}, \mathrm{t} \in \tilde{\mathrm{N}}$. From (a), we have

$\operatorname{Lim} n \rightarrow \infty \mathrm{M}\left(\mathrm{P}^{\mathrm{p}}\left(\mathrm{S}^{\mathrm{s}} x_{2 \mathrm{n}}\right), \mathrm{S}^{\mathrm{s}}\left(\mathrm{P}^{\mathrm{p}} x_{2 \mathrm{n}}\right), \frac{t}{2}\right)=1$ 
$\operatorname{Lim} n \rightarrow \infty \mathrm{N}\left(\mathrm{P}^{\mathrm{p}}\left(\mathrm{S}^{\mathrm{s}} x_{2 \mathrm{n}}\right), \mathrm{S}^{\mathrm{s}}\left(\mathrm{P}_{x 2 \mathrm{n}}^{\mathrm{p}}\right) \frac{t}{2}\right)=0$

Hence,

$\mathrm{M}\left(\mathrm{S}^{\mathrm{s}}\left(\mathrm{P}^{\mathrm{p}} x_{2 \mathrm{n}}\right), \mathrm{S}^{\mathrm{s}}(x), \mathrm{t}\right) \geq \min \left\{\mathrm{M}\left(\mathrm{P}^{\mathrm{p}}\left(\mathrm{S}^{\mathrm{s}} x_{2 \mathrm{n}}\right), \mathrm{S}^{\mathrm{s}}\left(\mathrm{P}^{\mathrm{p}} x_{2 \mathrm{n}}\right), \frac{t}{2}\right), \mathrm{M}\left(\mathrm{S}^{\mathrm{s}} \mathrm{P}^{\mathrm{p}}\left(x_{2 \mathrm{n}}\right), \mathrm{S}^{\mathrm{s}} x, \frac{t}{2}\right)\right\}$

$>1-\varepsilon$,

$\mathrm{N}\left(\mathrm{S}^{\mathrm{s}}\left(\mathrm{P}^{\mathrm{p}} x_{2 \mathrm{n}}\right), \mathrm{S}^{\mathrm{s}}(x), \mathrm{t}\right) \leq \max \left\{\mathrm{N}\left(\mathrm{P}^{\mathrm{p}}\left(\mathrm{S}^{\mathrm{s}} x_{2 \mathrm{n}}\right), \mathrm{S}^{\mathrm{s}}\left(\mathrm{P}^{\mathrm{p}} x_{2 \mathrm{n}}\right), \frac{\boldsymbol{t}}{2}\right), \mathrm{N}\left(\mathrm{S}^{\mathrm{S}} \mathrm{P}^{\mathrm{p}}\left(x_{2 \mathrm{n}}\right), \mathrm{S}^{\mathrm{s}} x, \frac{\boldsymbol{t}}{2}\right)\right\}$

$<\varepsilon$

for all $\mathrm{n} \geq$ no.

Therefore $\lim n \rightarrow \infty\left(\mathrm{P}^{\mathrm{p}} \mathrm{S}^{\mathrm{s}} x_{2 \mathrm{n}}\right),=\mathrm{S}^{\mathrm{s}} x$.

Also since $\lim n \rightarrow \infty \mathrm{Q}^{\mathrm{q}} x_{2 \mathrm{n}-1}={ }_{x}$ and $\mathrm{T}$ is continuous,

$\lim n \rightarrow \infty \mathrm{T}^{\mathrm{t}}\left(\mathrm{Q}^{\mathrm{q} x 2 \mathrm{n}-1}\right)=\mathrm{T}^{\mathrm{t} x}$.

Thus for $\mathrm{t}>0$ and $\varepsilon \in(0,1)$, there exists an $\mathrm{n}_{\mathrm{o}} \in \tilde{\mathrm{N}}$ such that

$\mathrm{M}\left(\mathrm{T}^{\mathrm{t}}\left(\mathrm{Q}_{x 2 \mathrm{n}-1}^{\mathrm{q}}\right), \mathrm{T}^{\mathrm{t}}(x), \mathrm{t} / 2\right)>1-\varepsilon, \mathrm{N}\left(\mathrm{T}^{\mathrm{t}}\left(\mathrm{Q}_{x 2 \mathrm{n}-1}^{\mathrm{q}}\right), \mathrm{T}^{\mathrm{t}}(x), \mathrm{t} / 2\right)<\varepsilon$

for all $\mathrm{n} \geq \mathrm{n}_{\mathrm{o}}$.

From (a), We have

Hence

$$
\begin{aligned}
& \lim n \rightarrow \infty \mathrm{M}\left(\mathrm{Q}^{\mathrm{q}}\left(\mathrm{T}^{\mathrm{t}} x_{2 \mathrm{n}-1}\right), \mathrm{T}^{\mathrm{t}}\left(\mathrm{Q}^{\mathrm{q}} x_{2 \mathrm{n}-1}\right), \mathrm{t} / 2\right)=1 \\
& \lim n \rightarrow \infty \mathrm{N}\left(\mathrm{Q}^{\mathrm{q}}\left(\mathrm{T}^{\mathrm{t}} x_{2 \mathrm{n}-1}\right), \mathrm{T}^{\mathrm{t}}\left(\mathrm{Q}^{\mathrm{q}} x_{2 \mathrm{n}-1}\right), \mathrm{t} / 2\right)=0
\end{aligned}
$$

$$
\begin{aligned}
\mathrm{M}\left(\mathrm{Q}^{\mathrm{q}}\left(\mathrm{T}^{\mathrm{t}} x_{2 \mathrm{n}-1}\right), \mathrm{T}^{\mathrm{t}} x, \mathrm{t}\right) & \geq \min \left\{\mathrm{M}\left(\mathrm{Q}^{\mathrm{q}}\left(\mathrm{T}^{\mathrm{t}} x_{2 \mathrm{n}-1}\right), \mathrm{T}^{\mathrm{t}}\left(\mathrm{Q}^{\mathrm{q}} x_{2 \mathrm{n}-1}\right), \mathrm{t} / 2\right), \mathrm{M}\left(\mathrm{T}^{\mathrm{t}}\left(\mathrm{Q}^{\mathrm{q}} x_{2 \mathrm{n}-1}\right), \mathrm{T}^{\mathrm{t}} x, \mathrm{t}\right)\right\} \\
& \geq 1-\varepsilon \\
\mathrm{N}\left(\mathrm{Q}^{\mathrm{q}}\left(\mathrm{T}^{\mathrm{t}} x_{2 \mathrm{n}-1}\right), \mathrm{T}^{\mathrm{t}} x, \mathrm{t}\right) & \leq \max \left\{\mathrm{N}\left(\mathrm{Q}^{\mathrm{q}}\left(\mathrm{T}^{\mathrm{t}} x_{2 \mathrm{n}-1}\right), \mathrm{T}^{\mathrm{t}}\left(\mathrm{Q}^{\mathrm{q}} x_{2 \mathrm{n}-1}\right), \mathrm{t} / 2\right), \mathrm{N}\left(\mathrm{T}^{\mathrm{t}}\left(\mathrm{Q}^{\mathrm{q}} x_{2 \mathrm{n}-1}\right), \mathrm{T}^{\mathrm{t}} x, \mathrm{t}\right)\right\} \\
& \leq \varepsilon
\end{aligned}
$$

for all $\mathrm{n} \geq \mathrm{n}_{\mathrm{o}}$,

Therefore $\lim n \rightarrow \infty \mathrm{Q}^{\mathrm{q}}\left(\mathrm{T}^{\mathrm{t}} x_{2 \mathrm{n}-1}\right)=\mathrm{T}^{\mathrm{t}} x$.

Using (d) with $\alpha=1$, we have

$\mathrm{M}\left(\mathrm{P}^{\mathrm{P}}\left(\mathrm{S}^{\mathrm{s}} x_{2 \mathrm{n}}\right), \mathrm{Q}^{\mathrm{q}}\left(\mathrm{T}^{\mathrm{t}} x_{2 \mathrm{n}-1}\right), \mathrm{K} \mathrm{t}\right) \geq \operatorname{Min}\left\{\mathrm{M}\left(\mathrm{S}^{\mathrm{s}}\left(\mathrm{S}^{\mathrm{s}} x_{2 \mathrm{n}}\right), \mathrm{T}^{\mathrm{t}}\left(\mathrm{T}^{\mathrm{t}} x_{2 \mathrm{n}-1}\right), \mathrm{t}\right), \mathrm{M}\left(\mathrm{P}^{\mathrm{p}}\left(\mathrm{S}^{\mathrm{s}} x_{2 \mathrm{n}}\right), \mathrm{S}^{\mathrm{s}}\left(\mathrm{S}^{\mathrm{s}} x_{2 \mathrm{n}}\right), \mathrm{t}\right)\right.$,

$\mathrm{M}\left(\mathrm{Q}^{\mathrm{q}}\left(\mathrm{T}^{\mathrm{t}} x_{2 \mathrm{n}-1}\right), \mathrm{T}^{\mathrm{t}}\left(\mathrm{T}^{\mathrm{t}} x_{2 \mathrm{n}-1}\right), \mathrm{t}\right), \mathrm{M}\left(\mathrm{P}^{\mathrm{p}}\left(\mathrm{S}^{\mathrm{s}} x_{2 \mathrm{n}}\right), \mathrm{T}^{\mathrm{t}}\left(\mathrm{T}^{\mathrm{t}} x_{2 \mathrm{n}-1}\right), \mathrm{t}\right), \quad \mathrm{M}\left(\mathrm{Q}^{\mathrm{q}}\left(\mathrm{T}^{\mathrm{t}} x_{2 \mathrm{n}-1}\right), \mathrm{S}^{\mathrm{s}}\left(\mathrm{S}^{\mathrm{s}}\right.\right.$ $\left.\left.\left.x_{2 \mathrm{n}}\right), \mathrm{t}\right)\right\}$

$\mathrm{N}\left(\mathrm{P}^{\mathrm{p}}\left(\mathrm{S}^{\mathrm{s}} x_{2 \mathrm{n}}\right), \mathrm{Q}^{\mathrm{q}}\left(\mathrm{T}^{\mathrm{t}} x_{2 \mathrm{n}-1}\right), \mathrm{K} \mathrm{t}\right) \leq \operatorname{Max}\left\{\mathrm{N}\left(\mathrm{S}^{\mathrm{s}}\left(\mathrm{S}^{\mathrm{s}} x_{2 \mathrm{n}}\right), \mathrm{T}^{\mathrm{t}}\left(\mathrm{T}^{\mathrm{t}} x_{2 \mathrm{n}-1}\right), \mathrm{t}\right), \mathrm{N}\left(\mathrm{P}^{\mathrm{p}}\left(\mathrm{S}^{\mathrm{s}} x_{2 \mathrm{n}}\right), \mathrm{S}^{\mathrm{s}}\left(\mathrm{S}^{\mathrm{s}} x_{2 \mathrm{n}}\right), \mathrm{t}\right)\right.$,

$\mathrm{N}\left(\mathrm{Q}^{\mathrm{q}}\left(\mathrm{T}^{\mathrm{t}} x_{2 \mathrm{n}-1}\right), \mathrm{T}^{\mathrm{t}}\left(\mathrm{T}^{\mathrm{t}} x_{2 \mathrm{n}-1}\right), \mathrm{t}\right), \mathrm{N}\left(\mathrm{P}^{\mathrm{P}}\left(\mathrm{S}^{\mathrm{s}} x_{2 \mathrm{n}}\right), \mathrm{T}^{\mathrm{t}}\left(\mathrm{T}^{\mathrm{t}} x_{2 \mathrm{n}-1}\right), \mathrm{t}\right), \quad \mathrm{N}\left(\mathrm{Q}^{\mathrm{q}}\left(\mathrm{T}^{\mathrm{t}} x_{2 \mathrm{n}-1}\right), \mathrm{S}^{\mathrm{s}}\left(\mathrm{S}^{\mathrm{s}}\right.\right.$ $\left.\left.\left.x_{2 \mathrm{n}}\right), \mathrm{t}\right)\right\}$

Taking limit as $n \rightarrow \infty$ and Using above results,

$\mathrm{M}\left(\mathrm{S}^{\mathrm{s}} x, \mathrm{~T}^{\mathrm{t}} x^{\mathrm{K}} \mathrm{t}\right) \geq \min \left\{\mathrm{M}\left(\mathrm{S}^{\mathrm{s}} x, \mathrm{~T}^{\mathrm{t}} x, \mathrm{t}\right), \mathrm{M}\left(\mathrm{S}^{\mathrm{s}} x, \mathrm{~S}^{\mathrm{s}} x, \mathrm{t}\right), \mathrm{M}\left(\mathrm{T}_{x}^{\mathrm{t}}, \mathrm{T}_{x}^{\mathrm{t}}, \mathrm{t}\right)\right.$,

$\geq \mathrm{M}\left(\mathrm{S}_{x,}^{\mathrm{s}}, \mathrm{T}_{x}^{\mathrm{t}}, \mathrm{t}\right)$

$$
\left.\mathrm{M}\left(\mathrm{S}_{x}^{\mathrm{s}}, \mathrm{T}_{x}^{\mathrm{t}}, \mathrm{t}\right), \mathrm{M}\left(\mathrm{T}_{x}^{\mathrm{t}}, \mathrm{S}_{x}^{\mathrm{s}}, \mathrm{t}\right)\right\}
$$

$\mathrm{N}\left(\mathrm{S}_{x}^{\mathrm{s}}, \mathrm{T}_{x}^{\mathrm{t}}, \mathrm{K}^{\mathrm{t}}\right) \leq \operatorname{Max}\left\{\mathrm{N}\left(\mathrm{S}_{x}^{\mathrm{s}}, \mathrm{T}_{x}^{\mathrm{t}}, \mathrm{t}\right), \mathrm{N}\left(\mathrm{S}_{x}^{\mathrm{s}}, \mathrm{S}_{x}^{\mathrm{s}}, \mathrm{t}\right), \mathrm{N}\left(\mathrm{T}_{x}^{\mathrm{t}}, \mathrm{T}_{x}^{\mathrm{t}}, \mathrm{t}\right)\right.$,

$$
\left.\mathrm{N}\left(\mathrm{S}_{x}^{\mathrm{s}}, \mathrm{T}_{x}^{\mathrm{t}}, \mathrm{t}\right), \mathrm{N}\left(\mathrm{T}_{x}^{\mathrm{t}}, \mathrm{S}_{x,}^{\mathrm{s}}, \mathrm{t}\right)\right\}
$$

which implies $\quad \mathrm{S}_{x}^{\mathrm{s}}=\mathrm{T}_{x}^{\mathrm{t}}$.

Now from (d) with $\alpha=1$,

$\mathrm{M}\left(\mathrm{P}_{x}^{\mathrm{p}}, \mathrm{Q}^{\mathrm{q}}\left(\mathrm{T}^{\mathrm{t}} x_{2 \mathrm{n}-1}\right), \mathrm{kt}\right) \geq \min \left\{\mathrm{M}\left(\mathrm{S}_{x}^{\mathrm{s}}, \mathrm{T}^{\mathrm{t}}\left(\mathrm{T}^{\mathrm{t}} x_{2 \mathrm{n}-1}\right), \mathrm{t}\right), \mathrm{M}\left(\mathrm{P}^{\mathrm{p}} x, \mathrm{~S}_{\mathrm{x}}^{\mathrm{s}}, \mathrm{t}\right)\right.$,

$$
\left.\mathrm{M}\left(\mathrm{Q}^{\mathrm{q}}\left(\mathrm{T}^{\mathrm{t}} x_{2 \mathrm{n}-1}\right), \mathrm{T}^{\mathrm{t}}\left(\mathrm{T}^{\mathrm{t}} x_{2 \mathrm{n}-1}\right), \mathrm{t}\right), \mathrm{M}\left(\mathrm{P}_{x}^{\mathrm{p}}, \mathrm{T}^{\mathrm{t}}\left(\mathrm{T}^{\mathrm{t}} x_{2 \mathrm{n}-1}\right), \mathrm{t}\right), \mathrm{M}\left(\mathrm{Q}^{\mathrm{q}}\left(\mathrm{T}^{\mathrm{t}} x_{2 \mathrm{n}-1}\right), \mathrm{S}_{x}^{\mathrm{s}}, \mathrm{t}\right)\right\}
$$

$\mathrm{N}\left(\mathrm{P}_{x}^{\mathrm{p}}, \mathrm{Q}^{\mathrm{q}}\left(\mathrm{T}^{\mathrm{t}} x_{2 \mathrm{n}-1}\right), \mathrm{kt}\right) \leq \max \left\{\mathrm{N}\left(\mathrm{S}_{x}^{\mathrm{s}}, \mathrm{T}^{\mathrm{t}}\left(\mathrm{T}^{\mathrm{t}} x_{2 \mathrm{n}-1}\right), \mathrm{t}\right), \mathrm{N}\left(\mathrm{P}_{x}^{\mathrm{p}}, \mathrm{S}_{\mathrm{x}}^{\mathrm{s}}, \mathrm{t}\right)\right.$,

$$
\left.\mathrm{N}\left(\mathrm{Q}^{\mathrm{q}}\left(\mathrm{T}^{\mathrm{t}} x_{2 \mathrm{n}-1}\right), \mathrm{T}^{\mathrm{t}}\left(\mathrm{T}^{\mathrm{t}} x_{2 \mathrm{n}-1}\right), \mathrm{t}\right), \mathrm{N}\left(\mathrm{P}_{x}^{\mathrm{p}}{ }^{\mathrm{T}} \mathrm{T}^{\mathrm{t}}\left(\mathrm{T}^{\mathrm{t}} x_{2 \mathrm{n}-1}\right), \mathrm{t}\right), \mathrm{N}\left(\mathrm{Q}^{\mathrm{q}}\left(\mathrm{T}^{\mathrm{t}} x_{2 \mathrm{n}-1}\right), \mathrm{S}_{x}^{\mathrm{s}} \mathrm{t}\right)\right\}
$$

Taking the limit as $n \rightarrow \infty$ and using above results

$\mathrm{M}\left(\mathrm{P}_{x}^{\mathrm{p}}, \mathrm{T}_{x}^{\mathrm{t}}, \mathrm{K} \mathrm{t}\right) \geq \min \left\{\mathrm{M}\left(\mathrm{T}_{x}^{\mathrm{t}}, \mathrm{T}_{x}^{\mathrm{t}} \mathrm{t}\right), \mathrm{M}\left(\mathrm{P}_{x}^{\mathrm{p}}, \mathrm{T}_{x}^{\mathrm{t}}, \mathrm{t}\right), \mathrm{M}\left(\mathrm{T}_{x}^{\mathrm{t}}, \mathrm{T}_{x}^{\mathrm{t}}, \mathrm{t}\right), \mathrm{M}\left(\mathrm{P}_{x}^{\mathrm{p}} \mathrm{T}_{x}^{\mathrm{t}}{ }^{\mathrm{t}}\right), \mathrm{M}\left(\mathrm{T}_{x}^{\mathrm{t}}, \mathrm{T}_{x}^{\mathrm{t}}{ }^{\mathrm{t}}\right)\right\}$,

$$
\geq \mathrm{M}\left(\mathrm{P}_{x}^{\mathrm{p}}, \mathrm{T}_{x}^{\mathrm{t}}, \mathrm{t}\right)
$$

$\mathrm{N}\left(\mathrm{P}_{x}^{\mathrm{p}}{ }_{x} \mathrm{~T}_{x}^{\mathrm{t}}, \mathrm{k} \mathrm{t}\right) \leq \max \left\{\mathrm{N}\left(\mathrm{T}_{x}^{\mathrm{t}}, \mathrm{T}_{x}^{\mathrm{t}}, \mathrm{t}\right), \mathrm{N}\left(\mathrm{P}_{x}^{\mathrm{p}}, \mathrm{T}_{x}^{\mathrm{t}}, \mathrm{t}\right), \mathrm{N}\left(\mathrm{T}_{x}^{\mathrm{t}}, \mathrm{T}_{x, \mathrm{t}}^{\mathrm{t}}\right), \mathrm{N}\left(\mathrm{P}_{x}^{\mathrm{p}}, \mathrm{T}_{x}^{\mathrm{t}}, \mathrm{t}\right), \mathrm{N}\left(\mathrm{T}_{x}^{\mathrm{t}} \mathrm{T}_{x}^{\mathrm{t}}, \mathrm{t}\right)\right\}$ 


$$
\leq \mathrm{N}\left(\mathrm{P}_{x}^{\mathrm{p}}, \mathrm{T}_{x}^{\mathrm{t}}, \mathrm{t}\right)
$$

Which implies $\mathrm{P}_{x}^{\mathrm{p}}=\mathrm{T}_{x}^{\mathrm{t}}$. Also since

$$
\mathrm{M}\left(\mathrm{P}_{x}^{\mathrm{p}}, \mathrm{Q}_{x}^{\mathrm{q}}, \mathrm{K} \mathrm{t}\right) \geq \mathrm{M}\left(\mathrm{P}_{x}^{\mathrm{p}}, \mathrm{Q}_{x}^{\mathrm{q}}, \mathrm{t}\right), \mathrm{N}\left(\mathrm{P}_{x}^{\mathrm{p}}, \mathrm{Q}_{x}^{\mathrm{q}}, \mathrm{kt}\right) \leq \mathrm{N}\left(\mathrm{P}^{\mathrm{p}}{ }_{x}, \mathrm{Q}_{x}^{\mathrm{q}}, \mathrm{t}\right)
$$

Hence $\mathrm{P}_{x=}^{\mathrm{p}}{ }_{x=}{ }^{\mathrm{Q} x}$. Therefore $\mathrm{P}^{\mathrm{p}}{ }_{x}=\mathrm{Q}_{x}^{\mathrm{q}}=\mathrm{S}_{x}^{\mathrm{s}}=\mathrm{T}_{x}^{\mathrm{t}}$.

Furthermore using (d) with $\alpha=1$, we have

$$
\begin{aligned}
& \mathrm{M}\left(\mathrm{P}_{x 2 \mathrm{n}}^{\mathrm{p}}, \mathrm{Q}_{x}^{\mathrm{q}}, \mathrm{K} \mathrm{t}\right) \geq \min \left\{\mathrm{M}\left(\mathrm{S}_{x^{2 \mathrm{n}}}^{\mathrm{s}}, \mathrm{T}_{x}^{\mathrm{t}}, \mathrm{t}\right), \mathrm{M}\left(\mathrm{P}_{x^{2 \mathrm{n}}}^{\mathrm{p}}, \mathrm{S}_{x^{2 \mathrm{n}}}^{\mathrm{s}}, \mathrm{t}\right), \mathrm{M}\left(\mathrm{Q}_{x}^{\mathrm{q}}, \mathrm{T}_{x}^{\mathrm{t}}, \mathrm{t}\right),\right. \\
& \left.\mathrm{M}\left(\mathrm{P}_{x^{2} \mathrm{n}}^{\mathrm{p}}, \mathrm{T}_{x, \mathrm{t}}^{\mathrm{t}} \mathrm{t}\right), \mathrm{M}\left(\mathrm{Q}_{x}^{\mathrm{q}}, \mathrm{S}_{x^{2 \mathrm{n}}, \mathrm{t}}^{\mathrm{S}}\right)\right\} \\
& \mathrm{N}\left(\mathrm{P}^{\mathrm{p}}{ }_{x 2 \mathrm{n}}, \mathrm{Q}_{x}^{\mathrm{q}}, \mathrm{K} \mathrm{t}\right) \leq \max \left\{\mathrm{N}\left(\mathrm{S}_{x^{2 \mathrm{n}}}^{\mathrm{s}}, \mathrm{T}_{x}^{\mathrm{t}}, \mathrm{t}\right), \mathrm{N}\left(\mathrm{P}_{x^{2 \mathrm{n}}}^{\mathrm{p}}, \mathrm{S}_{x^{2 \mathrm{n}}}^{\mathrm{s}}, \mathrm{t}\right), \mathrm{N}\left(\mathrm{Q}_{x}^{\mathrm{q}}, \mathrm{T}_{x}^{\mathrm{t}}, \mathrm{t}\right),\right. \\
& \left.\mathrm{N}\left(\mathrm{P}_{x^{2} \mathrm{n}}^{\mathrm{p}}, \mathrm{T}_{x}^{\mathrm{t}}, \mathrm{t}\right), \mathrm{N}\left(\mathrm{Q}_{x}^{\mathrm{q}}, \mathrm{S}_{x^{2 \mathrm{n}}}^{\mathrm{s}}, \mathrm{t}\right)\right\}
\end{aligned}
$$

Taking limit as $n \rightarrow \infty$ we have

$$
\begin{aligned}
\mathrm{M}\left(x, \mathrm{Q}_{x}^{\mathrm{q}}, \mathrm{K} \mathrm{t}\right) & \geq \min \left\{\mathrm{M}\left(x, \mathrm{Q}_{x}^{\mathrm{q}}, \mathrm{t}\right), \mathrm{M}\left(x, \mathrm{Q}_{x}^{\mathrm{q}}, \mathrm{t}\right), \mathrm{M}\left(\mathrm{Q}^{\mathrm{q}}{ }_{x}, \mathrm{Q}_{x}^{\mathrm{q}}, \mathrm{t}\right), \mathrm{M}\left(x, \mathrm{Q}_{x}^{\mathrm{q}}, \mathrm{t}\right), \mathrm{M}\left(\mathrm{Q}_{x}^{\mathrm{q}}, x, \mathrm{t}\right)\right\} \\
& \geq \mathrm{M}\left(x, \mathrm{Q}_{x}^{\mathrm{q}}, \mathrm{t}\right), \\
\mathrm{N}\left(x, \mathrm{Q}_{x}^{\mathrm{q}}, \mathrm{K} \mathrm{t}\right) & \leq \max \left\{\mathrm{N}\left(x, \mathrm{Q}_{x}^{\mathrm{q}}, \mathrm{t}\right), \mathrm{N}\left(x, \mathrm{Q}_{x}^{\mathrm{q}}, \mathrm{t}\right), \mathrm{N}\left(\mathrm{Q}_{x}^{\mathrm{q}}, \mathrm{Q}_{x}^{\mathrm{q}}, \mathrm{t}\right), \mathrm{N}\left(x, \mathrm{Q}_{x}^{\mathrm{q}}, \mathrm{t}\right), \mathrm{N}\left(\mathrm{Q}_{x}^{\mathrm{q}}, x, \mathrm{t}\right)\right\} \\
& \leq \mathrm{N}\left(x, \mathrm{Q}_{x}^{\mathrm{q}}, \mathrm{t}\right) .
\end{aligned}
$$

Which implies $x=\mathrm{Q}^{\mathrm{q}} x$

Therefore $x=\mathrm{Q}^{\mathrm{q}}{ }_{x}=\mathrm{P}^{\mathrm{p}}{ }_{x}=\mathrm{S}_{x}^{\mathrm{s}}=\mathrm{T}_{x}^{\mathrm{t}}$

That is, $x$ is a common fixed point of $\mathrm{P}^{\mathrm{p}}, \mathrm{Q}^{\mathrm{q}}, \mathrm{S}^{\mathrm{s}}$ and $\mathrm{T}^{\mathrm{t}}$. Let $\mathrm{z}$ be another common fixed point of maps. Then from (d) with $\alpha=1$

$$
\begin{aligned}
& M\left(p_{x}^{p}, Q^{q} z, k t\right) \geq \operatorname{Min}\left\{M\left(S_{x}^{s} T_{z}^{t}, t\right), M\left(P_{x}^{p}, S_{x}^{s}, t\right), M\left(Q_{z}^{q}, T_{z}^{t}, t\right),\right. \\
& \left.\mathrm{M}\left(\mathrm{P}_{x}^{\mathrm{p}}, \mathrm{T}^{\mathrm{t}} \mathrm{z}, \mathrm{t}\right), \mathrm{M}\left(\mathrm{Q}_{z}^{\mathrm{q}}, \mathrm{S}_{x}^{\mathrm{s}}, \mathrm{t}\right)\right\} \\
& \geq \operatorname{Min}\{\mathrm{M}(x, \mathrm{z}, \mathrm{t}), \mathrm{M}(x, x, \mathrm{t}), \mathrm{M}(\mathrm{z}, \mathrm{z}, \mathrm{t}), \mathrm{M}(x, \mathrm{z}, \mathrm{t}), \mathrm{M}(\mathrm{z}, x, \mathrm{t})\} \\
& \geq \mathrm{M}(x, \mathrm{z}, \mathrm{t}) \\
& \mathrm{N}\left(\mathrm{p}^{\mathrm{p}}{ }_{x}, \mathrm{Q}^{\mathrm{q}} \mathrm{z}, \mathrm{K} \mathrm{t}\right) \leq \operatorname{Max}\left\{\mathrm{N}\left(\mathrm{S}_{x}^{\mathrm{s}}, \mathrm{T}^{\mathrm{t}} \mathrm{z}, \mathrm{t}\right), \mathrm{N}\left(\mathrm{P}_{x}^{\mathrm{p}}, \mathrm{S}_{x}^{\mathrm{s}}, \mathrm{t}\right), \mathrm{N}\left(\mathrm{Q}_{\mathrm{z}}^{\mathrm{q}}, \mathrm{T}_{\mathrm{z}}^{\mathrm{t}}, \mathrm{t}\right),\right. \\
& \left.\mathrm{N}\left(\mathrm{P}_{x}^{\mathrm{p}}, \mathrm{T}_{\mathrm{z}}^{\mathrm{t}}, \mathrm{t}\right), \mathrm{N}\left(\mathrm{Q}_{\mathrm{z}}^{\mathrm{q}}, \mathrm{S}_{x}^{\mathrm{s}}, \mathrm{t}\right)\right\} \\
& \leq \operatorname{Max}\{\mathrm{N}(x, \mathrm{z}, \mathrm{t}), \mathrm{N}(x, x, \mathrm{t}), \mathrm{N}(\mathrm{z}, \mathrm{z}, \mathrm{t}), \mathrm{N}(x, \mathrm{z}, \mathrm{t}), \mathrm{N}(\mathrm{z}, x, \mathrm{t})\} \\
& \leq \quad \mathrm{N}(x, \mathrm{z}, \mathrm{t}) \\
& x=\mathrm{z} \text {. }
\end{aligned}
$$

Hence $x$ is a unique common fixed point of maps.

Third, we prove that this point $x$ is a common fixed point of $\mathrm{P}, \mathrm{Q}, \mathrm{S}$ and $\mathrm{T}$.

Since $\mathrm{P}_{x}=\mathrm{P}\left(\mathrm{P}_{x}^{\mathrm{p}}\right)=\mathrm{P}^{\mathrm{p}}\left(\mathrm{P}_{x}\right)$ and $\mathrm{P}_{x}=\mathrm{P}\left(\mathrm{S}_{x}^{\mathrm{s}}\right)=\mathrm{S}^{\mathrm{s}}\left(\mathrm{P}_{x}\right)$

from (a), hence $\mathrm{P}_{x}$ is a common fixed point of $\mathrm{P}^{\mathrm{p}}$ and $\mathrm{S}^{\mathrm{s}}$. Also since $\mathrm{Q}_{x}=\mathrm{Q}\left(\mathrm{Q}_{x}^{\mathrm{q}}\right)=\mathrm{Q}^{\mathrm{q}}\left(\mathrm{Q}_{x}\right)$ and $\mathrm{Q}_{x}-\mathrm{Q}\left(\mathrm{T}_{x}^{\mathrm{t}}\right)=$ $\mathrm{T}^{\mathrm{t}}\left(\mathrm{Q}_{x}\right)$ from (a), hence $\mathrm{Q}_{x}$ is a common fixed point of $\mathrm{Q}^{\mathrm{q}}$ and $\mathrm{T}^{\mathrm{t}}$. Now letting $x=\mathrm{P}_{x}$ and $\mathrm{y}=\mathrm{Q}_{x}$ and $\alpha=1$ in $(\mathrm{d})$, we have $\mathrm{M}\left(\mathrm{P} x, \mathrm{Q}_{x}, \mathrm{~K} \mathrm{t}\right)=\mathrm{M}\left(\mathrm{P}^{\mathrm{p}}\left(\mathrm{P}_{x}\right), \mathrm{Q}^{\mathrm{q}}\left(\mathrm{Q}_{x}\right), \mathrm{K} \mathrm{t}\right)$

$\geq \operatorname{Min}\left\{\mathrm{M}\left(\mathrm{S}^{\mathrm{s}}\left(\mathrm{P}_{x}\right), \mathrm{T}^{\mathrm{t}}(\mathrm{Q} x), \mathrm{t}\right), \mathrm{M}\left(\mathrm{P}^{\mathrm{P}}\left(\mathrm{P}_{x}\right), \mathrm{S}^{\mathrm{s}}\left(\mathrm{P}_{x}\right), \mathrm{t}\right)\right.$,

$\left.\mathrm{M}\left(\mathrm{Q}^{\mathrm{q}}\left(\mathrm{Q}_{x}\right), \mathrm{T}^{\mathrm{t}}\left(\mathrm{Q}_{x}\right), \mathrm{t}\right), \mathrm{M}\left(\mathrm{P}^{\mathrm{p}}\left(\mathrm{P}_{x}\right), \mathrm{T}^{\mathrm{t}}\left(\mathrm{Q}_{x}\right), \mathrm{t}\right), \mathrm{M}\left(\mathrm{Q}^{\mathrm{q}}\left(\mathrm{Q}_{x}\right), \mathrm{S}^{\mathrm{s}}\left(\mathrm{P}_{x}\right), \mathrm{t}\right)\right\}$

$=\operatorname{Min}\left\{\mathrm{M}\left(\mathrm{P}_{x}, \mathrm{Q}_{x}, \mathrm{t}\right), \mathrm{M}\left(\mathrm{P}_{x}, \mathrm{P}_{x}, \mathrm{t}\right), \mathrm{M}\left(\mathrm{Q}_{x}, \mathrm{Q}_{x}, \mathrm{t}\right), \mathrm{M}\left(\mathrm{P}_{x}, \mathrm{Q}_{x}, \mathrm{t}\right), \mathrm{M}\left(\mathrm{Q}_{x}, \mathrm{P}_{x}, \mathrm{t}\right)\right\}$

$\geq \mathrm{M}\left(\mathrm{P}_{x}, \mathrm{Q}_{x}, \mathrm{t}\right)$

$\mathrm{N}\left(\mathrm{P}_{x}, \mathrm{Q}_{x}, \mathrm{k} \mathrm{t}\right)=\mathrm{N}\left(\mathrm{P}^{\mathrm{p}}\left(\mathrm{P}_{x}\right), \mathrm{Q}^{\mathrm{q}}\left(\mathrm{Q}_{x}\right), \mathrm{K} \mathrm{t}\right)$

$\geq \operatorname{Max}\left\{\mathrm{N}\left(\mathrm{S}^{\mathrm{s}}\left(\mathrm{P}_{x}\right), \mathrm{T}^{\mathrm{t}}\left(\mathrm{Q}_{x}\right), \mathrm{t}\right), \mathrm{N}\left(\mathrm{P}^{\mathrm{p}}\left(\mathrm{P}_{x}\right), \mathrm{S}^{\mathrm{s}}\left(\mathrm{P}_{x}\right), \mathrm{t}\right), \mathrm{N}\left(\mathrm{Q}^{\mathrm{q}}\left(\mathrm{Q}_{x}\right), \mathrm{T}^{\mathrm{t}}\left(\mathrm{Q}_{x}\right), \mathrm{t}\right)\right.$,

$\left.\mathrm{N}\left(\mathrm{P}^{\mathrm{P}}\left(\mathrm{P}_{x}\right), \mathrm{T}^{\mathrm{t}}\left(\mathrm{Q}_{x}\right), \mathrm{t}\right), \mathrm{N}\left(\mathrm{Q}^{\mathrm{q}}\left(\mathrm{Q}_{x}\right), \mathrm{S}^{\mathrm{s}}\left(\mathrm{P}_{x}\right), \mathrm{t}\right)\right\}$

$=\operatorname{Max}\left\{\mathrm{N}\left(\mathrm{P}_{x}, \mathrm{Q}_{x}, \mathrm{t}\right), \mathrm{N}\left(\mathrm{P}_{x}, \mathrm{P}_{x}, \mathrm{t}\right), \mathrm{N}\left(\mathrm{Q}_{x}, \mathrm{Q}_{x}, \mathrm{t}\right), \mathrm{N}\left(\mathrm{P}_{x}, \mathrm{Q}_{x}, \mathrm{t}\right), \mathrm{N}\left(\mathrm{Q}_{x}, \mathrm{P}_{x}, \mathrm{t}\right)\right\}$

Therefore $\mathrm{P}_{x}=\mathrm{Q}_{x}$

$$
\geq \mathrm{N}\left(\mathrm{P}_{x}, \mathrm{Q}_{x}, \mathrm{t}\right)
$$

Also from (d) with $\alpha=1$, we have

$$
\begin{aligned}
\mathrm{M}\left(\mathrm{S}_{x}, \mathrm{~T}_{x}, \mathrm{~K} \mathrm{t}\right)= & \mathrm{M}\left(\mathrm{S}^{\mathrm{S}}\left(\mathrm{S}_{x}\right), \mathrm{T}^{\mathrm{t}}\left(\mathrm{T}_{x}\right), \mathrm{K} \mathrm{t}\right) \\
\geq & \operatorname{Min}\left\{\mathrm{M}\left(\mathrm{S}^{\mathrm{s}}\left(\mathrm{S}_{x}\right), \mathrm{T}^{\mathrm{t}}\left(\mathrm{T}_{x}\right), \mathrm{t}\right), \mathrm{M}\left(\mathrm{P}^{\mathrm{p}}\left(\mathrm{S}_{x}\right), \mathrm{S}^{\mathrm{s}}\left(\mathrm{S}_{x}\right), \mathrm{t}\right), \mathrm{M}\left(\mathrm{Q}^{\mathrm{q}}\left(\mathrm{T}_{x}\right), \mathrm{T}^{\mathrm{t}}\left(\mathrm{T}_{x}\right), \mathrm{t}\right),\right. \\
& \left.\mathrm{M}\left(\mathrm{P}^{\mathrm{p}}\left(\mathrm{S}_{x}\right), \mathrm{T}^{\mathrm{t}}\left(\mathrm{T}_{x}\right), \mathrm{t}\right), \mathrm{M}\left(\mathrm{Q}^{\mathrm{q}}\left(\mathrm{T}_{x}\right), \mathrm{S}^{\mathrm{s}}\left(\mathrm{S}_{x}\right), \mathrm{t}\right)\right\} \\
= & \operatorname{Min}\left\{\mathrm{M}\left(\mathrm{S}_{x}, \mathrm{~T}_{x}, \mathrm{t}\right), \mathrm{M}\left(\mathrm{S}_{x}, \mathrm{~S}_{x}, \mathrm{t}\right), \mathrm{M}\left(\mathrm{T}_{x}, \mathrm{~T}_{x}, \mathrm{t}\right), \mathrm{M}\left(\mathrm{S}_{x}, \mathrm{~T}_{x}, \mathrm{t}\right), \mathrm{M}\left(\mathrm{T}_{x}, \mathrm{~S}_{x}, \mathrm{t}\right)\right\} \\
\geq & \mathrm{M}\left(\mathrm{S}_{x}, \mathrm{~T}_{x}, \mathrm{t}\right) \\
\mathrm{N}\left(\mathrm{S}_{x}, \mathrm{~T}_{x}, \mathrm{Kt}\right)= & \mathrm{N}\left(\mathrm{S}^{\mathrm{s}}\left(\mathrm{S}_{x}\right), \mathrm{T}^{\mathrm{t}}\left(\mathrm{T}_{x}\right), \mathrm{Kt}\right) \\
\leq & \operatorname{Max}\left\{\mathrm{N}\left(\mathrm{S}^{\mathrm{s}}\left(\mathrm{S}_{x}\right), \mathrm{T}^{\mathrm{t}}\left(\mathrm{T}_{x}\right), \mathrm{t}\right), \mathrm{N}\left(\mathrm{P}^{\mathrm{p}}\left(\mathrm{S}_{x}\right), \mathrm{S}^{\mathrm{s}}\left(\mathrm{S}_{x}\right), \mathrm{t}\right), \mathrm{N}\left(\mathrm{Q}^{\mathrm{q}}\left(\mathrm{T}_{x}\right), \mathrm{T}^{\mathrm{t}}\left(\mathrm{T}_{x}\right), \mathrm{t}\right),\right. \\
& \left.\quad \mathrm{N}\left(\mathrm{P}^{\mathrm{p}}\left(\mathrm{S}_{x}\right), \mathrm{T}^{\mathrm{t}}\left(\mathrm{T}_{x}\right), \mathrm{t}\right), \mathrm{N}\left(\mathrm{Q}^{\mathrm{q}}\left(\mathrm{Tss}_{x}\right), \mathrm{S}^{\mathrm{s}}\left(\mathrm{S}_{x}\right), \mathrm{t}\right)\right\} \\
= & \operatorname{Max}\left\{\mathrm{N}\left(\mathrm{S}_{x}, \mathrm{~T}_{x}, \mathrm{t}\right), \mathrm{N}\left(\mathrm{S}_{x}, \mathrm{~S}_{x}, \mathrm{t}\right), \mathrm{N}\left(\mathrm{T}_{x}, \mathrm{~T}_{x}, \mathrm{t}\right), \mathrm{N}\left(\mathrm{S}_{x}, \mathrm{~T}_{x}, \mathrm{t}\right), \mathrm{N}\left(\mathrm{T}_{x}, \mathrm{~S}_{x}, \mathrm{t}\right)\right\}
\end{aligned}
$$


$\geq \mathrm{N}\left(\mathrm{S}_{x}, \mathrm{~T}_{x}, \mathrm{t}\right)$

Therefore, $\mathrm{S} x=\mathrm{T} x$. Since $x$ is a unique common fixed point of $\mathrm{P}^{\mathrm{p}}, \mathrm{Q}^{\mathrm{q}}, \mathrm{S}^{\mathrm{s}}, \mathrm{T}^{\mathrm{t}}$. Hence $\mathrm{P} x=\mathrm{Q} x$ is a common fixed points of $\mathrm{P}^{\mathrm{p}}, \mathrm{S}^{\mathrm{s}}$ and $\mathrm{S} x=\mathrm{T} x$ is a common fixed points of $\mathrm{Q}^{\mathrm{q}}, \mathrm{T}^{\mathrm{t}}$. Hence $x=\mathrm{P} x=\mathrm{Q} x=\mathrm{S} x=\mathrm{T} x$. That is, $x$ is common fixed point of $\mathrm{P}, \mathrm{Q}, \mathrm{S}$ and $\mathrm{T}$.

\section{References}

[1] S. Banach; Theorie des operations linearires, Monografje Mathematyczne., Warsaw 1932

[2] M. Grabiec; Fixed point in fuzzy metric spaces, Fuzzy Sets and Systems 27 (1988), 385-389.

[3] G. Jungck; Compatible mappings and common fixed points, Internat. J. Math. Math. Sci. 9 (1986), 779-791.

[4] G.Jungck, K.B. Moon and S. Park; Compatible mappings and common fixed point (2), Internat, J. Math. Math. Sci. 11 (1988), No. 2 , 285-288.

[5] J. Kramosil and J. Michalek; Fuzzy metric and statistical metric spaces, Kybernetica 11 (1975), 326-334.

[6] J. H. Park; Intuitionistic fuzzy metric spaces, Chaos Solitons \& Fractals 22 (2004), no. 5, 1039-1046.

[7] J.H. Park, J.S. Park, and Y.C. Kwun; A common fixed point theorem in the intuitionistic fuzzy metric space, Advances in Natural Comput. Data Mining (Proc. $2^{\text {nd }}$ ICNC and $3^{\text {rd }}$ FSKD) (2006), 293-300

[8] J.S. Park; On some results intuitionistic fuzzy metric space, J. Fixed Point Theory \& Appl. 3 (2008), No. 1, 39-48.

[9] J.S. Park and S. Y. Kim; A fixed point Theorem in a fuzzy m3etric space, F. J.M.S. 1 (1999), No. 6, $927-934$.

[10] J.S. Park and S. Y. Kim; Common fixed point theorem and example in intuitionistic fuzzy metric space, J.K.I.I.S. 18 (2008), no. 4 , 524-529.

[11] J.S. Park and Y.C. Kwun; Some fixed point theorems in the intuitionistic fuzzy metric spaces, F.J.M.S. 24 (2007), No. 2 , $227-239$.

[12] J.S. Park, Y.C. Kwun, and J.H. Park; A fixed point theorem in the intuitionistic fuzzy metric spaces, F.J.M.S. 16 (2005), No. 2, 137149.

[13] B. Schweizer and A. Sklar; Statititical metric spaces, Pacific J. Math. 10 (1960), no. 10, 314-334

[14] B. Singh and M.S. Chauhan; Common Fixed points of compatible maps in fuzzy metric spaces, Fuzzy Sets and Systems 115 (2000), 471-475.

[15] L.A. Zadeh; Fuzzy sets, Inform, and Control 8 (1965), 338-353. 